\title{
Response Characteristic of Cable Stayed Bridges under Static Loading and Due to the Earthquakes in Longitudinal Direction
}

\author{
H. Saidi ${ }^{1)}$, M. Al-Rawi ${ }^{2)}$, A.F. Ali ${ }^{2)}$ and A. Adda ${ }^{3)}$ \\ ${ }^{1), 3)}$ Faculty of Engineering, University of Sidi-Bel-Abbes, Algeria. E-Mail: hayatsaidi2009@yahoo.fr \\ 2) Faculty of Engineering, University of Baghdad, Iraq
}

\begin{abstract}
This paper presents the dynamic (earthquake response) analysis of cable stayed bridges under different types of static loading and due to longitudinal directions of earthquake base excitations. The deck and the tower of the bridge were idealized by discrete element idealization scheme (space frame element) with warping considered as a seventh degree of freedom. For comparison purposes, the discrete element with six degrees of freedom (warping neglected) were also used to model the structure under investigation.
\end{abstract}

The cables were modelled by the nonlinear truss elements.

It was found that the warping becomes of significant influence on the behaviour of the bridge deck only if the deck is acted upon by loading that is coupled with initial torsional moment.

KEYWORDS: Response characteristic, Cable stayed bridges, Static loading, Earthquakes, Longitudinal direction.

\section{UNDAMPED FREE VIBRATION ANALYSIS}

The equation of motion of any damped system is given by (Clough and Peneien, 1993).

$[M]\{\ddot{U}\}+[C]\{\dot{U}\}+[k]\{U\}=\{P(t)\}$

where $\{U\},\{\dot{U}\}$ and $\{\ddot{U}\}$ are the time-dependent displacement, velocity and acceleration vectors, respectively and $\{F(t)\}$ is the applied load vector.

The system is assumed to have classical damping. Thus, the damping matrix is of the form:

$\mathrm{C}=2 \xi_{\mathrm{i}} \omega_{\mathrm{i}} \mathrm{M}$

where $\xi_{\mathrm{i}}$ is the damping ratio corresponding to the mode (i).

Accepted for Publication on 15/9/2013. $\omega_{\mathbf{i}}$ is the natural angular velocity (or circular frequency) of the system which vibrates at the mode shape (i).

The equation of motion for free vibration undamped system can be obtained by omitting the damping matrix and the load vector from Eq. (1), such that:

$[\mathrm{M}]\{\ddot{\mathrm{U}}\}+[\mathrm{K}]\{\mathrm{U}\}=\{0\}$.

Also, the free vibration motion of the system is simple harmonic, which may be expressed as:

$U(t)=\Phi_{i} \sin \omega_{i} t$

in which:

$\Phi$ represents the mode shape of the system which does not change with time, but only the amplitude varies. 
The acceleration vector in free vibration will be:

$\ddot{U}(t)=-\omega_{i}^{2} \Phi_{i} \sin \omega_{i} t=-\omega_{i}^{2}\{U\}$.

Substituting equations (4) and (5) into equation

(3) results in:

$\omega_{i}^{2}[M] \Phi_{i} \sin \omega_{i} t+[K] \Phi_{i} \sin \omega_{i} \cdot t=\{0\}$.

Omitting the sine terms, this equation can be written as:

$\left[[\mathrm{K}]-\omega_{\mathrm{i}}^{2}[\mathrm{M}]\right] \Phi_{\mathrm{i}}=\{0\}$.

The nontrivial solution is possible only when:

$$
\left|[\mathrm{K}]-\omega_{i}^{2}[\mathrm{M}]\right|=0
$$

Eq. (8) is called the frequency equation of the system. Expanding this equation will give an algebraic equation of an $n^{\text {th }}$ degree known as the characteristic equation of the system (Paz, 1985). The $n$ roots of this equation $\left(\omega_{1}^{2} \omega_{2}^{2}\right.$ ..$\left.\omega_{n}^{2}\right)$ represent the square of the circular frequencies of $n$ modes of vibration (eigen values) which are possible in the system, while the corresponding $n$ eigen vectors of this eigen problem represent the $n$ mode shapes of vibration of the system. It is known that the amplitude of these mode shapes is arbitrary, it is customary to normalize them. The most often used normalizing procedure in computer programs for structural vibration analysis involves adjusting each mode shape.

$$
\left\{\Phi_{\mathrm{i}}\right\}^{\mathrm{T}}[\mathrm{M}]\left\{\Phi_{\mathrm{i}}\right\}=\mathrm{I}
$$

The mode shapes normalized in this fashion are said to be orthonormal relative to the mass matrix (Clough and Peneien,1993) or M-orthonormalized. Then:

$\left\{\Phi_{\mathrm{i}}\right\}^{\mathrm{T}}[\mathrm{K}]\left\{\Phi_{\mathrm{i}}\right\}=[\lambda]$
$\left\{\Phi_{\mathrm{i}}\right\}^{\mathrm{T}}[\mathrm{M}]\left\{\Phi_{\mathrm{i}}\right\}=[\mathrm{I}]$ where $\left[\Phi_{\mathrm{i}}\right]$ is a modal matrix whose columns are M-orthonormalized mode shapes, $[\lambda]$ is a diagonal matrix which stores the square of the circulars frequencies and $[\mathrm{I}]$ is an identity matrix.

So, it is important to realize that all solution methods are iterative in nature because, basically solving the eigen value problem:

$\mathrm{K} \Phi=\lambda \mathrm{M} \Phi$

is equivalent to calculating the roots of the polynomial $\mathrm{P}(\lambda)$, which has an order equal to the order of $\mathrm{K}$ and $\mathrm{M}$.

The general groups of solution methods are given in (Chardrupatla and Belegundu, 1991) as well as in (Bathe and Wilson, 1976).

\section{The Stiffness and Mass Matrices}

The formulation of the stiffness and mass matrices is based on approximate displacement functions. The deck and tower are modeled by a three-dimensional beam-column element with warping deflection and bimoment inertia as an additional degree-of-freedom (7 degrees of freedom per node), whereas cables are modeled as space truss. Transformation matrices used to relate local-global and master-slave are formulated to be suitable for the proposed elements.

\section{Earthquake Response Analysis}

The earthquake response analysis of cable stayed bridges when acted upon by ground motion is studied using the response spectrum procedure.

The accelerogram of al-hindya earthquake are considered, in the longitudinal, lateral and vertical directions, separately.

The longiudinal base excitation component produces axial motion of the bridge deck, whereas the lateral base excitation component produces lateral and torsional motion, and for vertical base excitation component, only vertical motion is produced. 


\section{Methods of Seismic Analysis}

In general, the response of a structure to seismic forces can be evaluated based on one of the following analytical procedures (Dowrick, 1977):

1. Equivalent static force analysis.

2. Dynamic analysis, including time domain and frequency domain.

For large or complex structures, the static methods of seismic analysis are not reliable because of the complexity of the vibrational modes and hence, the directions of the resulting inertia forces. Therefore, the structural response should be based on dynamic analysis (Dowrick, 1977).

$>$ Direct integration of the equations of motion based on step by step procedure.

$>$ Normal mode analysis.

$>$ Response spectrum technique.

\section{Equation of Motion}

The governing equation of motion for a structure when subject to a single component of a uniform ground motion is given by (Clough and Peneien, 1993):

$$
[M]\{\ddot{U}\}+[C]\{\dot{U}\}+[K]\{U\}=-[M]\{R\} \ddot{U}_{r g}
$$

where $\{R\}$ denotes an earthquake influence vector consisting of ones and zeros, where the ones correspond to the degree of freedom in the direction of the base excitation component, and zeros elsewhere.

Equation (13) can be transformed to the normal coordinates to give the $\mathrm{n}^{\text {th }}$ vibration mode of the structure; that is:

$\ddot{Y}_{n}+2 \xi \omega_{n} \dot{Y}_{n}+\omega_{n}^{2} Y_{n}=-\frac{l_{n r}}{M_{n}} \ddot{U}_{r g}(t)$

where

$M_{n}=\Phi_{n}^{T} M \Phi_{n}$ is the generalized mass at mode (n).

$l_{n r}=\Phi_{n}^{T} M R_{n}$ is the modal earthquake excitation in the r-direction $(\mathrm{r}=\mathrm{x}, \mathrm{y}, \mathrm{z})$.

$\ddot{U}_{r g}(t)$ is the time varying base acceleration component in the r-direction.

The solution of Eq. (14) may be written as:

$$
Y_{n, \max }=\frac{l_{n r}}{M_{n} \omega_{n}^{2}} S a(\xi, T) .
$$

The displacement $U_{n}$ is given by the product of the mode shape $\Phi_{n}$ and the generalized coordinate $\left(Y_{n}\right)$; hence the local displacements are :

$\left\{U_{n r}\right\}=\Phi_{n} \frac{l_{n}}{M_{n} \omega_{n}^{2}} S a(\xi, T)$.

The elastic forces $F s$ associated with the relative displacements can be obtained directly by premultiplying the relative displacement by the stiffness matrix, such that:

$$
F s(t)=K U(t)=K \Phi Y(t) .
$$

It is more convenient to express these forces in terms of the equivalent inertia forces developed in the undamped vibration (Clough and Peneien,1993) such that:

$$
\left\{F_{\text {snr }}\right\}=\omega_{n}^{2}[M]\left\{U_{n r}\right\}=[M]\left\{\Phi_{n}\right\} \frac{l_{n r}}{M_{n}} S_{a r}(\xi, T) .
$$

\section{Structural Response}

The maximum response cannot be obtained by merely adding the modal maxima because these maxima usually do not occur at the same time.

In most cases, when one mode achieves its maximum response, the other model responses are less than their individual maxima. Many methods are used to calculate the maximum response.

The square root of the sum of the squares of the model response is one of these methods and is used in the present study, thus the maximum total displacement and force are approximated by:

$U_{\max }=\sqrt{\left(U_{1}\right)^{2} \max +\left(U_{2}\right)^{2} \max +\cdots}$

$F s_{\max }=\sqrt{\left(F s_{1}\right)^{2} \max +\left(F s_{2}\right)^{2} \max +\cdots}$

where $\left(U_{1 \max }, U_{2 \max } \ldots\right)$ and $\left(F s_{1 \max }, F s_{2 \max } \ldots\right)$ are calculated from Eqs. (16) and (17), respectively. 


\section{Response Characteristic to Cable Stayed Bridges}

A cable stayed bridge is composed of the following: towers, girders, stays and foundations sustaining them. Such a kind of bridge can be regarded as a coupled vibrational system composed of these plural elements.

The coupled vibration in the superstructure is particular to cable stayed bridges, while the coupled vibration between superstructure and foundation is common to ordinary bridges.

The coupled phenomenon in the superstructure can be divided into two responses (Nojiri et al., 1977), as follows:

\section{Response Characteristic to Transverse Component of Earthquakes}

As stays are generally set in the longitudinal vertical plane which is perpendicular to both planes, where a tower or a girder makes response, the stiffening effects of stays are scarcely expected. Then, the main vibrating elements; i.e. the tower and the girder, are restricted by each other only on the pier, and consequently, the form of a so- called weakly coupled system.

In this case, the natural frequencies of this partial system are close, two corresponding modes also with close frequencies exist in the coupled system. One is like a superposition of each mode in partial systems with same phase; the other, with an inverse phase. These modes are deformed in comparison with modes in non-coupled system.

\section{Response Characteristic to Longitudinal Component of Earthquakes}

In this case, a tower and a girder vibrate in the same plane, in which stays are set tightly. Then, a tower and a girder form a strongly coupled system. It can be considered that the natural frequencies hardly come extremely close to each other and that the complex interaction between a tower and a girder as mentioned above rarely occurs.

\section{Case Study}

Al-Adhamiyah bridge whose configuration is shown in Figure 1 is studied for its earthquake response behavior when acted upon by the separate base excitations in three normal directions.
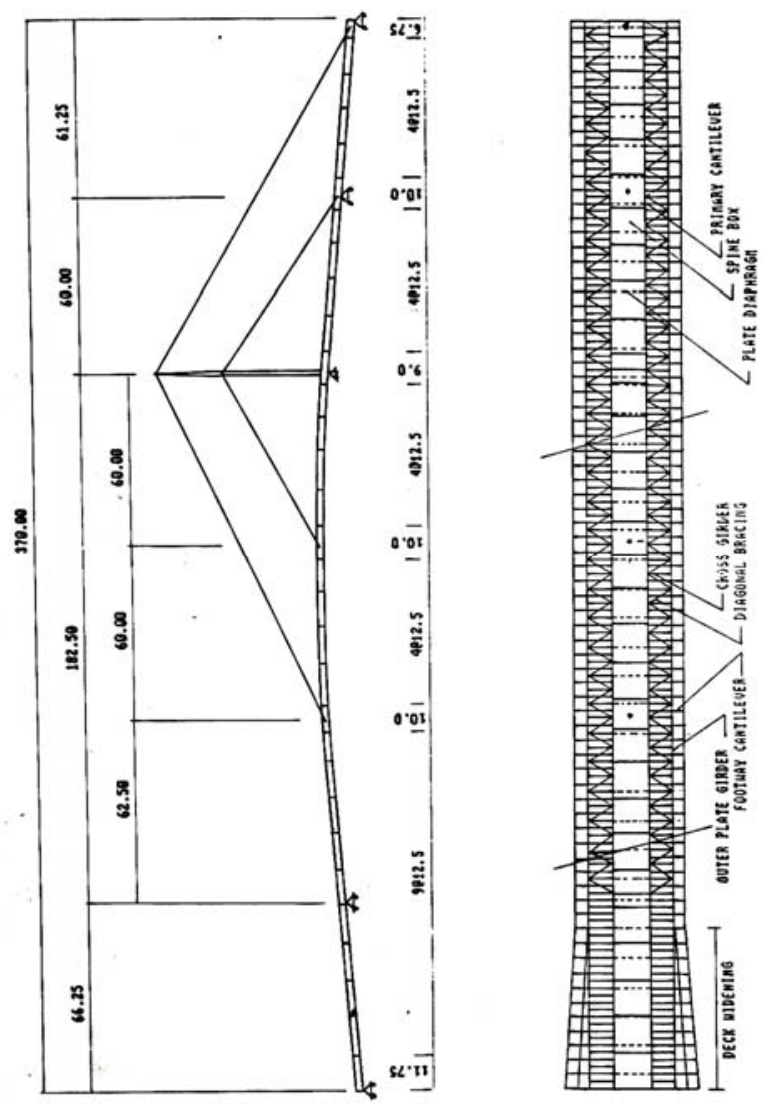

Figure (1): Al-Adhamiya bridge

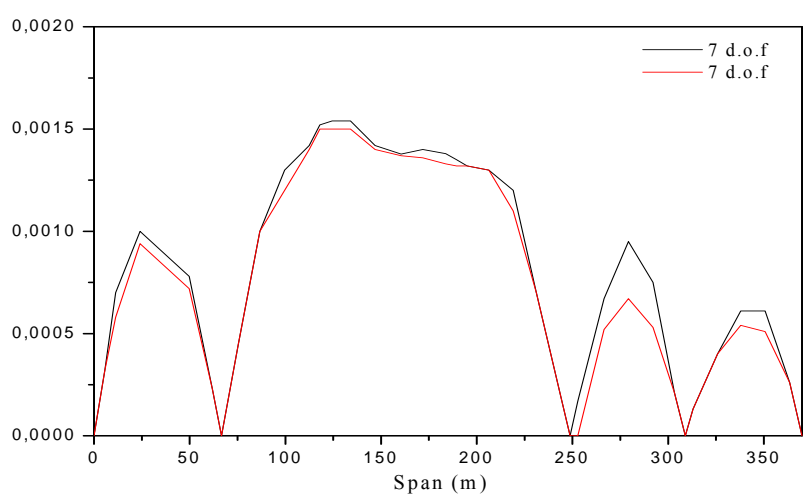

Figure (2): Maximum vertical displacement of the bridge deck under own and military loawhen the earthquake is in $\mathrm{X}$-direction (m) 


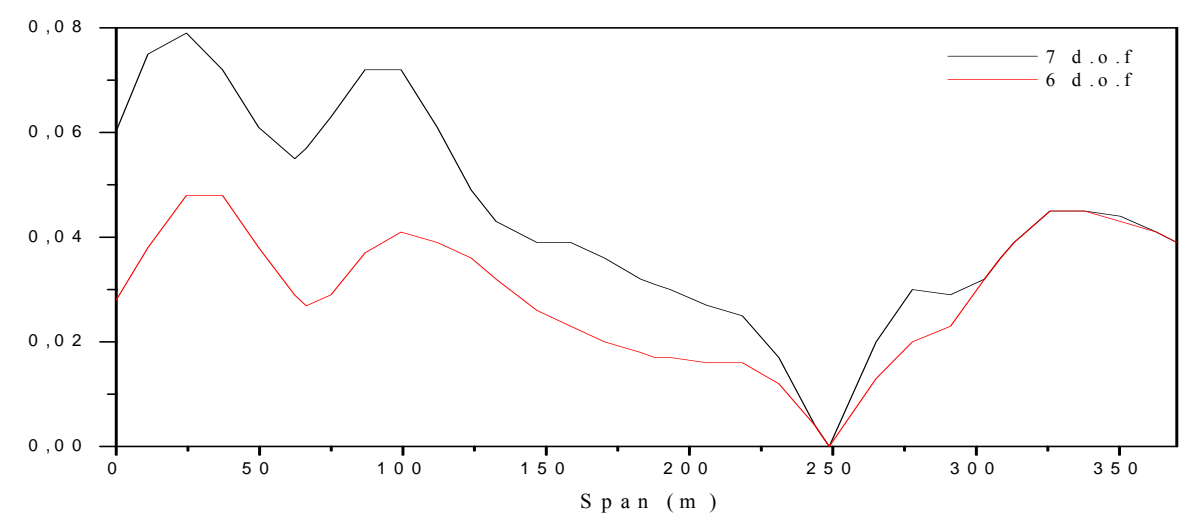

Figure (3): Maximum longitudinal displacement of the bridge deck under own and military load when the earthquake is in $\mathrm{X}$ - direction (m)

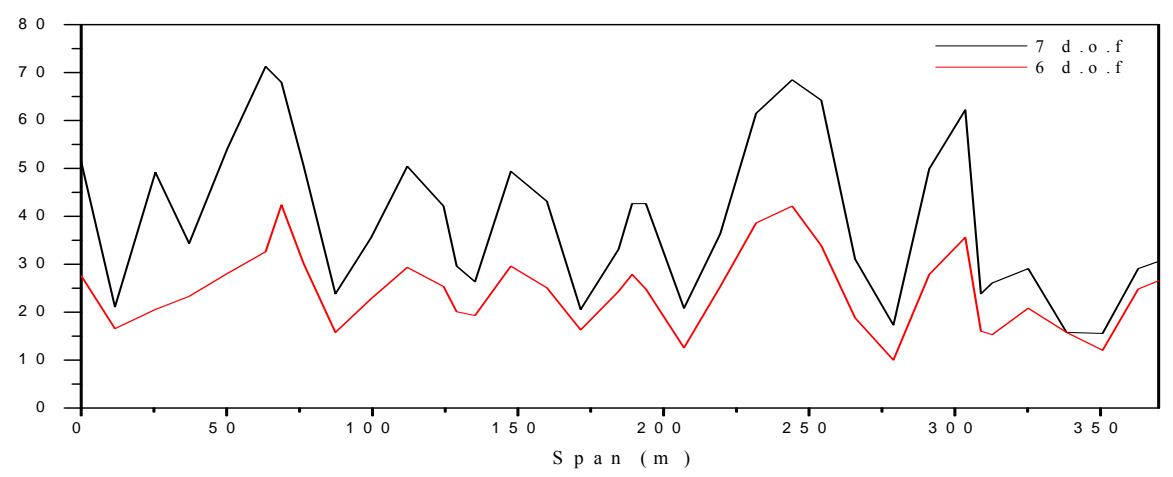

Figure (4): Maximum shear force of the bridge deck under own and military load when the earthquake is in $\mathrm{X}$-direction $(\mathrm{kN})$

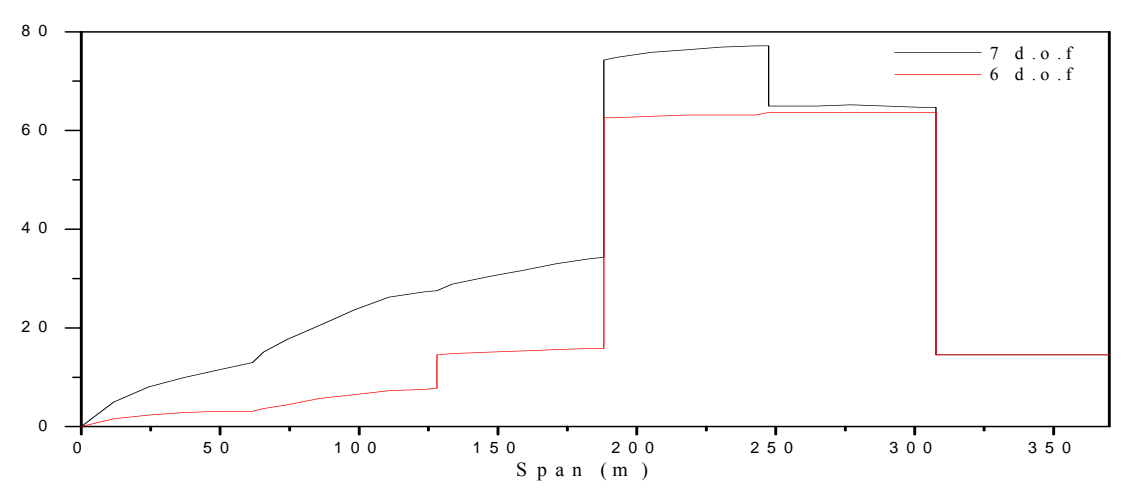

Figure (5): Maximum axial forces of the bridge deck under own and military load when the earthquake is in $\mathrm{X}$ - direction $(\mathrm{kN})$ 


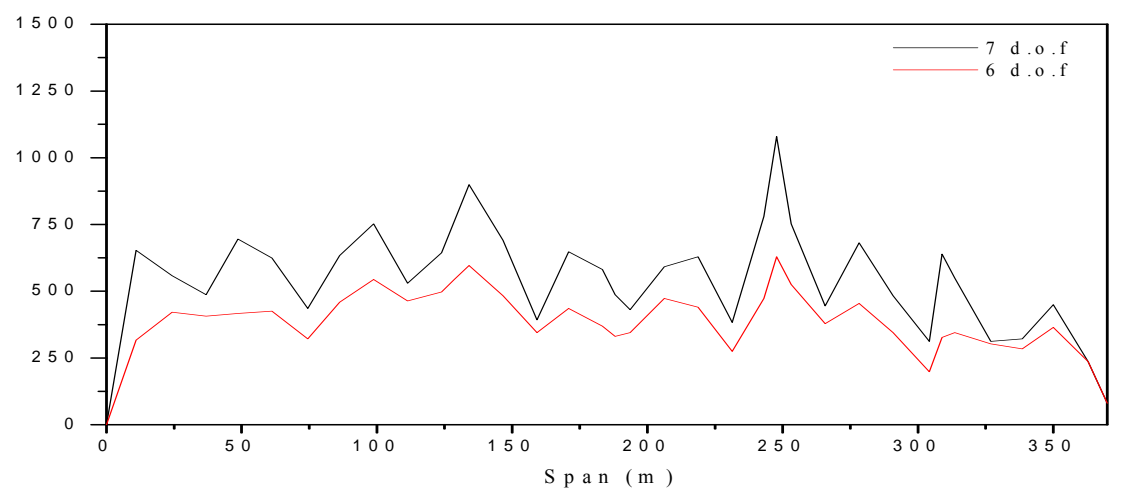

Figure (6): Maximum bending moment of the bridge deck under own and military load when the earthquake is in X-direction (kN.m)

\section{CONCLUSIONS}

The dynamic (earthquake response) analysis of cable stayed bridges under different types of static loading and due to the longitudinal directions of earthquake base excitations is carried out. The deck and the tower of the bridge were idealized by discrete element idealization scheme (space frame element) with warping considered as a seventh degree of freedom.

The comparison of the results is made vis-a-vis the discrete idealization approach with warping neglected; that is using 6 d.o.f. per node.

\section{REFERENCES}

Bathe, K.J., and Wilson, E.L. (1976). "Numerical Methods in Finite Element Analysis", Prentice-Hall, New Jersey.

Bathe, K. J., and Wilson, E.L. (1972). "Large Eigen Value Problems in Dynamic Analysis”. Jour. Eng. Mech. Div., ASCE, 98 (6), December, 1771-1483.

Chandrupatla, T.R., and Belegundu, A. (1991). "Introduction to Finite Element in Engineering". Prentice - Hall, New Delhi.

Clough, R. W., and Peneien, J. (1993). "Dynamics of Structures", McGraw-Hill, $13^{\text {th }}$ Printing, Singapore.
The plots presented for earthquake response of the bridge demonstrate that warping has significant influences on the structure response when the structure is acted upon by a base excitation in the longitudinal direction.

In the lateral response, when warping is considered; that is using 7 d.o.f. per node, the structure response seems to be larger than the response of the structure with warping neglected (6 d.o.f. per node), especially close to the tower base, this behavior is related to the increasing torsional and warping stiffness which results in large torsional response.

Dowrick, D.K. (1977). "Earthquake Resistant Design", John Wiley \& Sons, LTD, London.

Iraqi Standard Specification for Road Bridges (Loading). (1978). Ministry of Housing and Construction, Baghdad, Republic of Iraq.

Nojiri, Y. et al. (1977). "Earthquake Resistant Design of Prestressed Concrete Cable Stayed Bridges". Proceedings of the Sixth World Conference on Earthquake Engineering, New Delhi, Jan.

Paz, M. (1985). "Structural Dynamics-Theory and Computations", Van Nostrand Reinhold Co. 